\title{
Evaluación neurofisiológica y clínica en pacientes con diabetes mellitus
}

\author{
Roberto Portillo ${ }^{1,2}$, David Lira ${ }^{3}$, Miguel Quiñónez ${ }^{1,2}$
}

Resumen

\begin{abstract}
Objetivo: Determinar las características clínicas y hallazgos neurofisiológicos en pacientes diabéticos y correlacionar sus variables. Material y Métodos: Se evaluó 50 pacientes con diabetes mellitus del Hospital Nacional Guillermo Almenara. Se determinó edad, sexo, ocupación, tiempo de enfermedad, glicemia, dieta, tratamiento, sintomatología sensitiva, velocidad de conducción nerviosa motora y sensitiva de 12 nervios por paciente, 600 nervios en total, prevalencia de polineuropatía diabética (PND) y relacionamos las variables con la PND. Resultados: El $90 \%$ de pacientes presentó compromiso, $86 \%$ con criterios de PND; la edad osciló entre 38 y 78 años (media=58,5 años), 36 fueron varones (72\%), el tiempo de enfermedad promedio fue de 89 meses, la glicemia $143 \mathrm{mg} / \mathrm{dL}, 74 \%$ tenía dieta hipoglúcida, $48 \%$ refirió síntomas sensitivos. No hubo relación de PND con tiempo de enfermedad, glicemia, tratamiento o sintomatología sensitiva. Conclusiones: La exhaustiva anamnesis a pacientes diabéticos encontró 48\% de sintomatología sugestiva de PND, 86\% presentó hallazgos neurofisiológicos compatibles con PND, que no fue influida por otras variables; es imperativa la detección temprana de diabetes mellitus, para evitar complicaciones como la PND.
\end{abstract}

Palabras clave Diabetes mellitus; neuropatías diabéticas; neurofisiología; sensasión.

\section{Neurophysiological and clinical evaluation in patients with diabetes mellitus}

\begin{abstract}
Objective: To determine diabetic patients' clinical and neurophysiological characteristics and correlate variables. Material and Methods: Fifty diabetes mellitus patients were studied at Guillermo Almenara National Hospital. Age, sex, occupation, length of disease, glycaemia, diet, treatment, sensorial symptoms, motor (SMNC) and sensorial (SMNC) nerve conduction speed of 12 nerves by patient, total 600 nerves, and prevalence of diabetic neuropathy $(D N)$ were determined as well as the relation of the diverse variables with DN. Results: Ninety per cent of patients presented compromise, $86 \%$ fulfilled DN criteria; age was 38 to 78 years old ( mean $=58,5$ years), 36 were males (72\%), average time of disease was 89
\end{abstract}

1 Jefe del Servicio de Neurología de Hospital Guillermo Almenara, EsSalud. Lima, Perú.

2 Profesor Principal de la Facultad de Medicina de San Fernando, Universidad Nacional Mayor de San Marcos. Lima, Perú.

3 Profesor de la Facultad de Medicina de la Universidad San Martín de Porras. Lima, Perú. months, glycaemia was $143 \mathrm{mg} / \mathrm{dL}, 74 \%$ received hypoglycaemic diet, $48 \%$ referred sensorial symptoms. There was no relation between $D N$ and length of disease, glycaemia, treatment or sensorial symptoms. Conclusions: Exhaustive anamnesis to diabetic patients found $48 \%$ with DN suggestive symptoms, $86 \%$ presented neurophysiological findings compatible with DN; early detection of diabetes mellitus is important in order to avoid DN-like complications.

Key words: Diabetes mellitus; diabetic neuropathies; neurophysiology; sensation.

\section{INTRODUCCIÓN}

La diabetes mellitus (DM) es una de las enfermedades crónicas no transmisibles con mayor prevalencia en la población general a nivel mundial $\left({ }^{1}\right)$, que varía de 7,7 a $87,3 / 1000$ habitantes $\left({ }^{1-3}\right)$. Se caracteriza por un síndrome metabólico consistente en hiperglicemia, glucosuria, poliurea, polidipsia, polifagia y alteración en el metabolismo lipídico y proteico, 
como resultado de un déficit absoluto o relativo de acción de la insulina; esta alteración metabólica condiciona un daño progresivo e irreversible a diversos órganos y estructuras del organismo $\left({ }^{1}\right)$, siendo una de las complicaciones más frecuentes la polineuropatía diabética (PND), que se ha convertido en la primera causa de polineuropatía en el mundo occidental.

A la fecha, no existe un consenso que permita estandarizar el diagnóstico de la polineuropatía diabética, lo que no ha permitido determinar su incidencia y prevalencia. Los estudios realizados a la fecha muestran resultados que oscilan entre el 5 y $80 \%\left({ }^{4,5}\right)$, dependiendo de los criterios de selección de los pacientes, método de estudio empleado, formas clínicas consideradas, entre otras variables.

Otro problema aún sin definir totalmente es su fisiopatología. En base a los conocimientos médicos actuales, se considera tres teorías que permitirían entender el mecanismo de producción de la enfermedad, para orientar su tratamiento: de la vía poliol, de los productos finales de la glicosilación y la microvascular.

La teoría de la vía poliol se basa en el hecho de que en el nervio periférico (NP) el ingreso de glucosa no es dependiente de insulina, por lo que la hiperglicemia se acompaña de altos niveles de glucosa dentro del NP, que, mediante la enzima aldolasarreductasa, convierte a la glucosa en sorbitol y fructosa a través de la vía poliol, lo que asociado a la disminución de otro azúcar, el mioinositol, determinaría alteraciones estructurales y funcionales del NP $\left(^{6-9}\right)$.

En la teoría de los productos finales de la glicosilación, se considera que en los pacientes diabéticos la hiperglicemia determina mediante la reacción de Millard por la unión de aldolasas a los grupos NH libres de las proteínas y otras reacciones químicas la formación de productos finales de la glicosilación, que al unirse a sus receptores específicos activan a los monocitos y las células endoteliales aumentando el estrés oxidativo y produciendo alteraciones funcionales en el control del tono vascular, con lesiones microvasculares progresivas $\left({ }^{10-12}\right)$.

Mientras que la teoría microvascular considera que la irrigación del NP, que se realiza a través de los vasos sanguíneos epineurales y perineurales, que reciben inervación autonómica a través de un plexo que regula el flujo sanguíneo endoneural $\left({ }^{12}\right)$, podría verse comprometida en la DM, produciendo un compromiso progresivo de los nervios irrigados.

Algunos estudios han encontrado una disminución en la densidad del plexo perivascular, aún en pacientes que no habían desarrollado neuropatía, causando una alteración en la relajación vascular en los vasos epineurales, mostrando cambios en la microvasculatura del nervio, en la membrana basal, hiperplasia de células endoteliales y formación de puentes arteriovenosos; con reducción en la perfusión nerviosa e hipoxia endoneural $\left({ }^{12,13}\right)$.

Sin embargo, ninguna teoría por sí sola podría explicar las diversas manifestaciones clínicas de la PND y sus diferentes formas de presentación, pudiendo estar implicados varias o todas las teorías propuestas en su etiopatogenia.

Entidades más definidas como la neuropatía autonómica y la amiotrofia diabética, por la especificidad del daño y por la forma de progresión de la enfermedad implicaría mecanismos autoinmunes, por lo que sólo algunos diabéticos presentarían estos tipos de neuropatía $\left({ }^{14}\right)$.

Existen diversas clasificaciones de la PND $\left({ }^{15,16}\right)$, que consideran diversos criterios. Se presenta la clasificación propuesta por Dyck $\left({ }^{17}\right)$, sobre la base de diversos estadios de severidad, según criterios clínicos, estudios sensoriales y neurofisiológicos.

Los criterios para definir los estadios de polineuropatía diabética son:

I. Disminución de la velocidad de conducción nerviosa en 2 ó más de los nervios examinados. 
II. Elevación del umbral de percepción vibratoria y/o térmico en las manos y/o en los pies.

III. Disminución de la variabilidad de la frecuencia cardíaca en reposo o en la prueba de las 6 respiraciones profundas.

IV. Ausencia de los reflejos patelar y aquiliano, disminución de la fuerza muscular y/o trastornos de la sensibilidad.

V. Síntomas neuropáticos.

La polineuropatia diabetica es clasificada en:

- Estadio $O$ (sin neuropatía): menos de 2 hallazgos entre los criterios 1-4, con ausencia de síntomas neuropáticos.

- Estadio 1 (neuropatía subclínica = asintomática): 2 ó más hallazgos entre los criterios 1-4, con ausencia de síntomas neuropáticos.

- Estadio 2 (neuropatía sintomática): 2 ó más hallazgos entre los criterios 1-4 en presencia de síntomas neuropáticos $\left({ }^{17}\right)$.

El objetivo de nuestro estudio fue determinar las características clínicas y sintomatología sensitiva en pacientes diabéticos, la velocidad de conducción nerviosa motora (VCNM) y sensitiva (VCNS) de los nervios estudiados, la prevalencia de PND según criterios neurofisiológicos, así como determinar la correlación electrofisiológica y clínica en los pacientes con DM, para lo que sólo se consideró la sintomatología sensitiva y los estudios neurofisiológicos realizados.

\section{MATERIAL Y MÉTODOS}

Se realizó un estudio transversal de una serie consecutiva de pacientes con diagnóstico de DM, que acudían regularmente para tratamiento al servicio de endocrinología del Hospital Nacional Guillermo Almenara Irigoyen (HNGAI) de Lima, Perú, y que fueron referidos al servicio de neurología del HNGAI para evaluación, hasta completar 50 casos. El HNGAI es un hospital de cuarto nivel, siendo uno de los hospitales de mayor complejidad del sistema de seguridad social del Perú, que cubre aproximadamente a $24,1 \%$ de la población nacional; atiende de manera preferente pacientes referidos de establecimientos de menor nivel de complejidad, para diagnóstico definitivo y tratamiento especializado, teniendo como área de influencia una población de aproximadamente 1600000 habitantes en Lima y mayor población en el resto del Perú. Cuenta con equipos que permiten la realización de estudios neurofisiológicos, dentro de los que se considera la VCNM y VCNS.

Los sujetos de estudio fueron pacientes adultos de ambos sexos, con diagnóstico confirmado de DM. Se consideró como síntomas de PND la presencia de manifestaciones clínicas o subclínicas que evidenciaban una lesión o trastorno de la función de las estructuras del sistema nervioso periférico somático, que incluyen raíces espinales, craneales y ganglios sensitivos, mientras ocurran en relación aparente con la DM. A todos los sujetos de estudio se les realizó estudios de neuroconducción, los que debían de estar alterados en dos o más nervios periféricos para considerar la presencia de PND.

Se excluyó del estudio aquellos pacientes con diagnóstico de polineuropatía por otra causa diferente o concomitante a la PND y a aquellos que no desearon participar voluntariamente en el estudio.

Se llenó una ficha para recolección de los datos que consideró las variables demográficas, como edad en años, sexo, ocupación, así como los relacionados a la DM, como tiempo de enfermedad en meses, tratamiento al momento del estudio, glicemia sanguínea y el tipo de dieta que ingería. Asimismo, se determinó la presencia de sintomatología sensitiva, como dolor, parestesias, disestesias, entre otras, para lo que se realizó un interrogatorio al paciente en base a preguntas directas e individualizadas.

En una segunda fase, se realizó el estudio de la velocidad de conducción nerviosa periférica, 
tanto motora como sensitiva, en los siguientes nervios: mediano, cubital, radial, ciático poplíteo externo, tibial posterior y nervio facial derechos e izquierdos en cada uno de los pacientes, completando 600 nervios individuales. Para la determinación de las latencias y velocidad de conducción nerviosa motora y sensitiva, se empleó las técnicas estándar de acuerdo al nervio estudiado $\left({ }^{18}\right)$.

Se realizó el análisis de los datos y se confeccionó tablas de frecuencias y gráficos. Se calculó el porcentaje de pacientes con PND y se determinó su relación con la edad, sexo, tiempo de evolución de la DM, glicemia sanguínea, sintomatología sensitiva y medicación habitual y se determinó la existencia de diferencia estadística significativa mediante la aplicación de la prueba chi cuadrado. Todas las operaciones estadísticas han sido realizadas con el programa informático para análisis de datos SPSS 10,0 para Windows y Excel 2000 para Windows.

\section{RESULTADOS}

En los 50 pacientes estudiados, se realizó estudios neurofisiológicos en un total de 600 nervios individuales, que mostraron alteración en la VCNM y VCNS en $90 \%$ de los pacientes; los valores individuales evidenciaron $84 \%$ de compromiso del nervio mediano derecho, $66 \%$ del nervio cubital izquierdo, $6 \%$ del nervio radial bilateral, $54 \%$ del nervio ciático poplíteo externo, $52 \%$ del nervio tibial posterior y $50 \%$ del nervio facial, cuyos resultados según compromiso motor y sensitivo se detalla en la Tabla 1.

De acuerdo a la definición de PND, 43 pacientes $(86 \%)$ presentaron compromiso de 2 ó más nervios estudiados y cumplieron los criterios neurofisiológicos de PND; sólo 2 (4\%) tuvieron compromiso en un solo nervio, siendo catalogados como mononeuropatía y 5 pacientes $(10 \%)$ no presentaron alteración neurofisiológica (Tabla 2).
Con relación a las variables demográficas de la muestra, se encontró 36 varones $(72 \%)$ y 14 mujeres $(28 \%)$, con edades comprendidas entre los 38 y los 78 años de edad, con un promedio de 58,5 $\pm 10,6$ años, cuya distribución por grupos etáreos se presenta en la Figura 1; 45 pacientes tenían DM tipo II (90\%) y 5 DM tipo I (10\%). El tiempo desde el diagnóstico de la DM hasta el momento de la evaluación varió desde 1 hasta 240 meses, siendo en promedio de $89 \pm 69,4$ meses; $80 \%$ de los pacientes tenía 120 meses o menos de diagnóstico de DM al momento de la evaluación; los valores de glicemia sanguínea

Tabla 1. Estudio electrofisiológico de velocidad de conducción nerviosa motora (VCNM) y sensitiva (VCNS).

\begin{tabular}{|c|c|c|c|c|}
\hline \multirow[t]{2}{*}{ Nervio estudiado } & \multicolumn{2}{|c|}{ Derecho } & \multicolumn{2}{|c|}{ Izquierdo } \\
\hline & $\mathrm{n}$ & $\%$ & $\mathrm{n}$ & $\%$ \\
\hline 1.Mediano & 42 & 84 & 35 & 70 \\
\hline Compromiso motor & 36 & 72 & 28 & 56 \\
\hline Compromiso sensitivo & 1 & 2 & 2 & 4 \\
\hline Compromiso mixto & 5 & 10 & 5 & 10 \\
\hline Normal & 8 & 16 & 15 & 30 \\
\hline 2.Cubital & 31 & 62 & 33 & 66 \\
\hline Compromiso motor & 19 & 38 & 19 & 38 \\
\hline Compromiso sensitivo & 3 & 6 & 5 & 10 \\
\hline Compromiso mixto & 9 & 18 & 9 & 18 \\
\hline Normal & 19 & 38 & 17 & 34 \\
\hline \multicolumn{5}{|l|}{ 3.Radial } \\
\hline Compromiso sensitivo & 6 & 12 & 6 & 12 \\
\hline Normal & 44 & 88 & 44 & 88 \\
\hline \multicolumn{5}{|l|}{ 4. Ciático poplíteo externo } \\
\hline Compromiso motor & 20 & 40 & 27 & 54 \\
\hline Normal & 30 & 60 & 23 & 46 \\
\hline \multicolumn{5}{|l|}{ 5. Tibial poterior } \\
\hline Compromiso motor & 24 & 48 & 26 & 52 \\
\hline Normal & 26 & 52 & 24 & 48 \\
\hline \multicolumn{5}{|l|}{ 6. Facial } \\
\hline Compromiso motor & 23 & 46 & 25 & 50 \\
\hline Normal & 27 & 54 & 25 & 50 \\
\hline Total de nervios por lado & 300 & & 300 & \\
\hline
\end{tabular}


Tabla 2. Pacientes diabéticos según tipo de compromiso neurofisiológico.

\begin{tabular}{lcc}
\hline Número de nervios comprometidos & $\mathrm{n}$ & $\%$ \\
\hline Polineuropatía diabética $^{1}$ & 43 & 86 \\
Mononeuropatía $^{2}$ & 2 & 4 \\
Ningún nervio $_{\text {Total }}$ & 5 & 10 \\
& 50 & 100 \\
\hline
\end{tabular}

${ }^{1}$ Compromiso de 2 ó más nervios

${ }^{2}$ Compromiso de un solo nervio

fueron 64 a $320 \mathrm{mg} / \mathrm{dL}$, con un valor promedio de $143 \pm 54,3 \mathrm{mg} / \mathrm{dL} ; 37$ pacientes $(74 \%)$ tenían una dieta hipoglúcida, 11 (22\%) una dieta mixta y sólo $2(4 \%)$ una dieta normal. Los pacientes que refirieron síntomas sensitivos como dolor, parestesias, disestesias, entre otras molestias, fueron $24(48 \%)$, mientras que $26(52 \%)$ no refirieron molestias; todas las características de la muestra estudiada se resume en la Tabla 3 .

El número de pacientes con PND neurofisiológica, afectación de dos o más nervios estudiados, que presentaron sintomatología previa fue de $21(87,5 \%)$, mientras que 22 pacientes $(84,6 \%)$ no presentaron sintomatología previa, no existiendo diferencia estadística significativa $(p<0,01)$. Cuando se evaluó la prevalencia de PND en pacientes con DM insulinodependiente y con DM no insulinodependiente, no se encontró diferencia

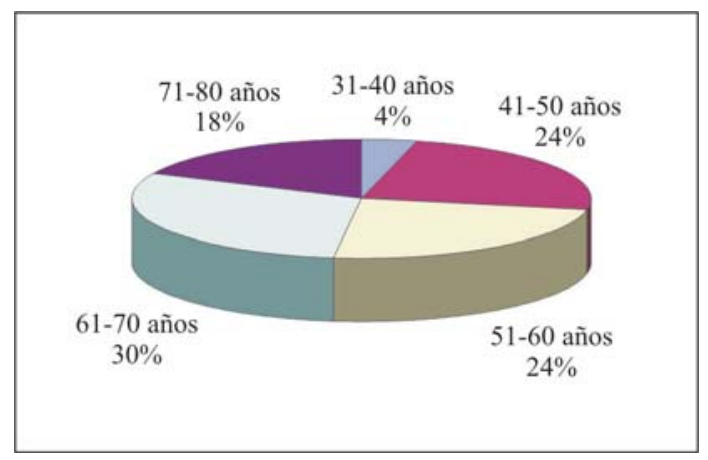

Figura 1. Pacientes diabéticos según grupos etáreos. estadística significativa. Cuando se analizó la edad, sexo, glicemia sanguínea y dieta según la presencia o no de PND, no se encontró diferencia estadística significativa $(p<0,01)$. Los 7 pacientes que no presentaban PND tenían un tiempo de evolución de la DM menor a 10 años, mientras que todos los pacientes que presentaron más de 10 años de evolución de la DM presentaron PND. Sin embargo, por el reducido tamaño del grupo de pacientes sin PND no se encontró diferencia estadística significativa $(p<0,01)$.

Tabla 3. Características de la muestra de pacientes diabéticos.

\begin{tabular}{|c|c|c|}
\hline & $\mathrm{n}$ & $\%$ \\
\hline \multicolumn{3}{|l|}{ Sexo } \\
\hline Masculino & 36 & 72 \\
\hline Femenino & 14 & 28 \\
\hline \multicolumn{3}{|l|}{ Tiempo de enfermedad } \\
\hline$<1$ año & 4 & 8 \\
\hline 1-10 años & 36 & 72 \\
\hline 10-20 años & 10 & 20 \\
\hline \multicolumn{3}{|c|}{ Glicemia al momento de la evaluación } \\
\hline$<120 \mathrm{mg} / \mathrm{dL}$ & 22 & 44 \\
\hline $121-200$ mg/dL & 21 & 42 \\
\hline $201-250$ mg/dL & 4 & 8 \\
\hline $251-300$ mg/dL & 2 & 4 \\
\hline $301-350 \mathrm{mg} / \mathrm{dL}$ & 1 & 2 \\
\hline \multicolumn{3}{|l|}{ Dieta } \\
\hline Hipoglúcida & 37 & 74 \\
\hline Mixta & 11 & 22 \\
\hline Normal & 2 & 4 \\
\hline \multicolumn{3}{|l|}{ Tratamiento actual } \\
\hline Sólo dieta & 10 & 20 \\
\hline Dieta y ADO & 34 & 68 \\
\hline Dieta, ADO e insulina & 1 & 2 \\
\hline Dieta e insulina & 5 & 10 \\
\hline \multicolumn{3}{|l|}{ Sintomatología referida } \\
\hline Dolor & 13 & 26 \\
\hline Parestesias & 11 & 22 \\
\hline Asintomáticos & 26 & 52 \\
\hline Total de la muestra & 50 & 100 \\
\hline
\end{tabular}

ADO: Antidiabéticos orales. 


\section{DISCUSIÓN}

El estudio electrofisiológico de los 600 nervios estudiados evidencia alteración en la VCNM y VCNS en el $90 \%$ de los pacientes estudiados, llegando $86 \%$ a cumplir criterios de PND, cifra superior a la reportada en otras series que muestran valores de hasta $80 \%\left({ }^{4,5}\right)$. Este alto porcentaje de compromiso podría deberse a que se consideró la realización del examen neurofisiológico de 12 nervios en cada uno de los pacientes estudiados, lo que aumenta la probabilidad de encontrar compromiso de 2 ó más nervios evaluados, ya que estudios similares consideran 6 a 8 nervios para la determinación de PND $\left({ }^{19}\right)$.

Nuestro estudio encontró un mayor compromiso motor de los nervios de las extremidades superiores: mediano derecho $82 \%$ y cubital izquierdo $66 \%$, en relación a las extremidades inferiores: tibial posterior izquierdo $52 \%$ y ciático poplíteo externo izquierdo $54 \%$, que contrasta con estudios similares que reportan una mayor afectación de las extremidades inferiores en estos pacientes $\left({ }^{19-21}\right)$. Nuestro estudio sólo evalúo la VCNS en las extremidades inferiores, por lo que no pudo ser comparado con otros estudios que reportan compromiso sensitivo simétrico en las 4 extremidades $\left({ }^{20-22}\right)$.

La distribución de la población por grupos etáreos muestra una distribución relativamente homogénea, excepto en el grupo de 31 a 40 años, que es más reducida; lo que podría deberse al hecho de que en su mayoría se trata de pacientes con DM no dependientes de insulina, secundarias al sobrepeso, variable que guarda una relación directa con la edad, aunque esta variable de manera independiente no guarda relación con la prevalencia de PND $\left({ }^{23,24}\right)$.

El tiempo de evolución de la enfermedad de los pacientes en nuestro estudio es amplio y no muestra diferencia estadística significativa en relación a la prevalencia de PND, lo que ha sido reportado por otros autores como una variable directa $\left({ }^{25}\right)$.
De la muestra estudiada, sólo $44 \%$ de los pacientes presenta valores de glicemia dentro de límites normales: $56 \%$ presenta valores altos, que han sido relacionados a mayor prevalencia de complicaciones crónicas dentro de las que se considera la PND $\left({ }^{26,27}\right)$, lo que podría explicar el alto porcentaje encontrado en nuestro estudio $(86 \%)$.

Cuando se evalúa el tipo de dieta, $26 \%$ de los pacientes no sigue una dieta hipoglúcida estricta, lo que estaría condicionando altos niveles de glicemia, con todas sus consecuencias a corto y largo plazo $\left({ }^{26}\right)$.

La indicación del tratamiento de los pacientes de la muestra con 10 pacientes que son controlados con sólo dieta al parecer se estaría cumpliendo a cabalidad, al presentar sólo uno de dichos pacientes una glicemia ligeramente alta $(150 \mathrm{mg} / \mathrm{dL})$, mientras que los 9 restantes presentan valores de glicemia normales. Cuando se evalúa la prevalencia de PND de acuerdo al tipo de dieta, no existe diferencia estadística significativa $(p<0,01)$, resultado que concuerda con otros estudios reportados en Australia y Canadá $\left({ }^{28,29}\right)$. Aunque algunos autores han relacionado el uso de insulina como factor de riesgo para el desarrollo de PND $\left({ }^{30}\right)$, lo que también ha sido observado en nuestro estudio, ya que todos los pacientes que recibían como tratamiento sólo insulina o asociada a antidiabéticos orales presentaron PND.

Ninguno de los pacientes incluidos en nuestro estudio fue previamente referido a nuestro servicio por su médico para evaluación por sintomatología sugestiva. Sin embargo, después de un interrogatorio minucioso 24 pacientes $(48 \%)$ refirieron presentar algún tipo de síntoma sugestivo de PND, de lo que resalta la importancia de la anamnesis en la valoración de estos pacientes.

Es interesante notar que sólo 5 pacientes (10\%) no presentaron alteración en los estudios neurofisiológicos realizados, lo que demuestra la alta prevalencia de la PND en los pacientes 
diabéticos presenten o no síntomas sugestivos de padecer ésta complicación crónica, resultado similar al reportado por Gómez-Viera et al $\left({ }^{22}\right)$, hallazgos que se correlacionan directamente con el compromiso neuropatológico descrito en otros estudios $\left({ }^{31}\right)$.

Cuando evaluamos la prevalencia de PND de acuerdo al tiempo de evolución de la enfermedad, si bien no se encontró diferencia estadística significativa por el reducido número de pacientes que no presentaron PND (16\%), se encontró que la presentaban todos los pacientes que tenían más de 10 años de enfermedad, resultados también reportados en otros estudios que encuentran una relación directa entre la prevalencia de PND y el tiempo de enfermedad de la DM $\left({ }^{2,20,23,25}\right)$. Gómez-Viera y col reportaron el descontrol metabólico de la DM y el tiempo de enfermedad mayor a 10 años como relacionados de manera significativa con la PND (22).

La alta prevalencia de PND encontrada en nuestro estudio evidencia su importancia como la más frecuente complicación crónica en los pacientes con DM, con el incremento en los gastos de medicamentos, atención médica especializada y discapacidad a largo plazo que conlleva; ya que muchas veces la DM es diagnosticada años después de haberse iniciado, debido a que estos pacientes presentan síntomas escasos o nulos. Por ello se hace imperativo incrementar la realización de controles anuales en la población asegurada que permita detectar a tiempo el inicio de esta enfermedad, mediante la implementación de campañas de educación y prevención sanitaria. Y una vez detectada, iniciar tratamiento precoz. tanto dietético como medicamentoso, que evite la aparición de las complicaciones tardías.

En base a los resultados obtenidos podemos concluir que cuando se realiza una exhaustiva anamnesis a los pacientes diabéticos se encuentra un alto porcentaje $(48 \%)$ con sintomatología sugestiva de PND que no se había detectado inicialmente; que la gran mayoría de pacientes diabéticos (86\%) presenta hallazgos neurofisiológicos compatibles con PND, no existiendo diferencia estadística significativa cuando se consideró la sintomatología previa, glicemia o tratamiento. Por lo que consideramos imperativo la realización de exámenes periódicos para la detección temprana de pacientes con DM, para evitar el desarrollo de complicaciones crónicas como la PND, que una vez instaladas se tornan irrversibles.

\section{REFERENCIAS BIBLIOGRÁFICAS}

1. Figuerola D, Reynals E. Diabetes mellitus. En: Farreras P, Rozman C. Medicina Interna. Madrid: Harcourt Brace de España S.A.; 1997.

2. Li G, Hu Y, Pan X. Prevalence and incidence of NIDDM in Daqing City. Chin Med J (Engl). 1996;9:599-602.

3. Edelstein SL, Knowler WC, Bain RP, et al. Predictors of progression from impaired glucose tolerance to NIDDM: an analysis of six prospective studies. Diabetes. 1997;46:701-10.

4. Franklin GM, Kahn LB, Baxter J. Sensory neuropathy in non-insulin-dependent diabetes mellitus. The San Valley Diabetes Study. Am J Epidemiol. 1990;131-43.

5. Maser RE, Steenkiste AR, Dorman JS. Epidemiological correlates of diabetic neuropathy. Report from Pittisburgh Epidemiology of Diabetes Complications Study. Diabetes. 1989;38:1456-61.

6. Greene DA, Sima AAF, Stevens MJ, Feldman EL, Lattimer SA. Complications: neuropathy, pathogenic considerations. Diabetes Care. 1992;15:1902-25.

7. Fisher SK, Heacock AM, Agranoff BW. Inositol lipids and signal transductions in the nervous system: an update. J Neurochem. 1992;58:18-38.

8. Del Monte MA, Rabbani R, Diaz TC, Lattimer SA. Sorbitol, myo-inositol and rod outer segment phagacytosis in glucoseexposed cultures human retinal pigment epithelial cells: in vitro modeling of the myo-inositol deplection hypothesis of diabetic complications. Diabetes. 1991;40:1335-45.

9. Romero J, Licea M. Patogénesis de la polineuropatía diabética. Rev Cub Endoc. 1996;7:1.

10. Bucola R, Tracey KJ, Cerami A. Advanced glycosylation products quench nitric oxide synthase and mediate defective endothelium-dependent vasodilatation in experimental diabetes. J Clin Invest. 1991;87:432-8.

11. Wautier JL, Guillausseau PJ. Advanced glycation end products, their receptors and diabetic angiopathy. Diabetes Metab. 2001;27:535-42.

12. Otero-Siliceo E, Ruano-Calderón A. Neuropatía diabética: ¿Una enfermedad vascular?. Rev Neurol. 2003;37:658-61.

13. Coppey LJ, Gellett JS, Davidson EP, Dunlap JA, Yorek MA. Changes in endoneural blood flow, motor nerve 
conduction velocity and vascular relaxation of epineural arterioles of the sciatic nerve in ZDF-obese diabetic rats. Diabetes Metab Res Rev. 2002;18:49-56.

14. Awada A, Dehoux E, Al Jumah M, Al Ayafi H. Rapidly evolving diabetic mononeuritis multiplex. Favorable outcome after immunosuppressive treatment. Rev Neurol (Paris). 2001;157:1427-9.

15. Valk JJP, Nauta RLM, Strijer RLM, Bertelsmann FW. Clinical examinations versus neurophysiological examination in the diagnosis of diabetic polyneuropathy. Diabet Med. 1992;9:716-21.

16. Dyck PJ, Karnes JL, O’Brien PC, Litchy WJ, Low PA, Melton III LJ. The Rochester Diabetic Neuropathy Study: reassessment of test and criteria for diagnosis and staged severity. Neurology. 1992;42:1164-70.

17. Dyck PJ. Detection, characterization, and staging of polyneuropathy: assessed in diabetics. Muscle Nerve. 1988;11:21-32.

18. Brown WF. The physiological and technical basis of electromyography. Boston: Butterworth; 1984.

19. Hendriksen P, Oey P, Wieneke G, Bravenboer B, van Huffelen C. Subclinical diabetic polyneuropathy: early detection of involvement of different nerve fibre types. J Neurol Neurosurg Psychiatry. 1993;56:509-14.

20. García-Inerárity H, Romero-Mestre J, Gómez-Fernández L, Mustelier-Béquer R, Torres-Govin G. Neuropatía autonómica y neuropatía somática en el paciente diabético: análisis electroclínico. Rev Neurol. 1999;29:1138-41.

21. Santiago S, Espinosa ML, Pérez-Conde MC, Merino M, Ferrer T. Afectación de fibras finas en la patología del nervio periférico. Rev Neurol. 1999;28:543-54.

22. Gómez-Viera N, Soto-Lavastida A, Roselló-Silva H, Gómez de Molina-Iglesias M. Factores de riesgo de la neuropatía diabética simétrica distal. Rev Neurol. 2001;32:806-12.

23. Valensi P, Giroux C, Seeboth-Ghalayini B, Attalis JR. Diabetic peripheral neuropathy: effects of age duration of diabetes, glycemic control, and vascular factors. J Diabetes Complications. 1997;11:27-34.

24. Summerson JH, Spangler JG, Bell RA, Shelton BJ, Konen JC. Association of gender with symptoms and complications in type 2 diabetes mellitus. Womens Health Issues. 1999;9:176-82.

25. Partanen J, Niskanen L, Lehtinen J. Natural history of peripheral neuropathy in patients with noninsulindependent diabetes mellitus. N Engl J Med. 1995;333:89-94.

26. Diabetes Control and Complications Trial Research Group. The effect of intensive treatment of diabetes on the development and progression of longterm complications in insulindependent diabetes mellitus. N Engl J Med. 1993;329:977-96.

27. Nathan DM. The pathophysiology of diabetic complications: How much does the glucose hypothesis explain? Ann Inter Med. 1996;124:90-6.

28. De Wytt CN, Jackson RV, Hockings GI, Joyner JM, Strakosch CR. Polyneuropathy in Australian outpatients with type 2 diabetes mellitus. J Diabetes Complications. 1999;13:74-8.

29. Tkac Y, Bril V. Glycemic control is related to the electrophysiologic severity of diabetic peripheral sensorimotor polyneuropathy. Diabetes Care. 1998;21:174952.

30. Shazly M, Abdel-Fattah M, Scorpiglione N, Benedetti MM, Capani F, Carinci F, et al. Risk factors for lower limb complications in diabetic patients. J Diabetes Complications. 1998; 12:10-7.

31. Behse F, Buchthal F, Carlsen F. Nerve biopsy and conduction studies in diabetic neuropathy. J Neurol Neurosurg Psychiatry. 1977;40:1072-82.

Manuscrito recibido el 05 de enero de 2005 y aceptado para publicación el 15 marzo 2005.

Correspondencia: Dr. David Lira Mamani

Servicio de Neurología, Hospital Nacional Guillermo Almenara Irigoyen Av. Grau 800. Lima 1, Perú.

Correo-e: davidliraaqp@hotmail.com 